\title{
Development of a Roebel-cable-based cos $\vartheta$ dipole: design and windability of magnet ends
}

\author{
Clément Lorin, Maria Durante, Philippe Fazilleau, Glyn Kirby, Lucio Rossi
}

\begin{abstract}
In the scope of the Future Circular Collider, work package 10, namely "Future Magnets", of the EuCARD2 project aims at investigating accelerator quality magnets made of High Temperature Superconductors. The present paper deals with development of a $\cos \vartheta$ dipole relying on Roebel cable technology. In the first part, we present in details the design of the dipole: the magnet generates $5 \mathrm{~T}$ in stand-alone mode with an overall current density of $684 \mathrm{~A} / \mathrm{mm}^{2}$. When operating in a background field of $13 \mathrm{~T}$ an extra field of $2 \mathrm{~T}$ can be provided by the $\cos \vartheta$ insert due to mechanical limitations. A field increase up to $2.5 \mathrm{~T}$ is conceivable providing the addition of inner shell reinforcement that would reduce the magnet aperture from $40 \mathrm{~mm}$ to $30 \mathrm{~mm}$. In the second part, winding tests of dummy Roebel cable are reported. They demonstrate the challenges stemming from the relative slippage of the cable tapes while winding that may be overcome by a lengthening of the longitudinal gap of the cable either by an increase of the transposition pitch or a decrease of the number of tapes in the cable. The cable shows a smooth winding behavior along the coil for two different magnet end designs as well as for its path outwards the magnet.
\end{abstract}

Index Terms-Superconducting dipole magnet, HTS Roebel cable, accelerator quality magnet, EuCARD2

\section{INTRODUCTION}

$\mathrm{I}^{\mathrm{N}}$ $\mathrm{N}$ the near future, high energy physics, among others, may require very high magnetic field magnets [1]. Targeting magnetic fields beyond $16 \mathrm{~T}$ requires a technological jump towards High Field Superconductors (HFS), commonly named High Temperature Superconductors (HTS). Indeed, Low Temperature Superconductors (LTS) such as $\mathrm{Nb}-\mathrm{Ti}$ or $\mathrm{Nb}_{3} \mathrm{Sn}$ do not allow for generating magnetic fields larger than $15 \mathrm{~T}$. Both technologies - LTS and HFS - must be coupled into one hybrid magnet where only its core, subjected to the highest field, would consist of HFS, the outer windings being made of LTS. Therefore, in the framework of two European Commission funded projects, namely EuCARD-WP7 [2] and EuCARD2-WP10 [3][4], application of HFS to accelerator magnet inserts has been developed. Progress about the EuCARD-WP7 HFS racetrack insert can be found in [5]. As for the EuCARD2 project, it focuses on a ten kA-class HTS cable and on a dipole insert for which various coil

Manuscript received October 12, 2015.

C. Lorin, M. Durante and P. Fazilleau are with CEA Saclay, France (Clement.Lorin@gmail.com). G. Kirby and L. Rossi are with Cern, Switzerland. EuCARD-2 is co-funded by the partners and the European Commission under Capacities 7th Framework Programme, Grant Agreement 312453. configurations have been investigated: block design with stack tapes [6], or with Roebel cable [7] - currently under construction [8] - as well as $\cos \vartheta$ insert design made of Roebel cable. This paper is a follow-up of the latter for which previous work has showed that a classical double-layer configuration of the $\cos \vartheta$ insert would lead to non-bearable stress in the winding [9]. In short, the weakness of the mechanical structure - to fit in the aperture of the LTS outsert magnet - would result in an ovalization of the HFS magnet pinching the median coil block. Therefore, a single-layer configuration with a thicker external structure has been investigated and is described hereafter. A first part presents electromagnetic and mechanical 2D models of the dipole insert. Then a section is dedicated to the 3D analysis of both the end designs and the shape of the innermost turn that goes out the magnet. Finally winding test results of the 3D end design with a stainless-steel dummy Roebel cable are reported.

\section{2D SINGLE LAYER COS- $\Theta$ DESIGN}

In stand-alone operation, a central field up to $5 \mathrm{~T}$ has to be produced by the HTS insert in a 40 -mm-diameter aperture. The insert will eventually be inserted inside a $100-\mathrm{mm}-$ diameter aperture $\mathrm{Nb}_{3} \mathrm{Sn}$ magnet with a $13 \mathrm{~T}$ central field at $4.2 \mathrm{~K}$, called FRESCA2 [10], to increase its field by about $2.5 \mathrm{~T}$. The magnet will be wound with a $10 \mathrm{kA}$ class Roebel cable which is a continuously transposed cable [11]. The Roebel cable assembly will be made at the Karlsruhe Institute of Technology with RE-BCO tapes to be provided by Bruker HTS [12]. The main features of the baseline cable are shown in Table I and Fig. 1. The cable is insulated with a glass fiber sleeve of $100 \mu \mathrm{m}$ in thickness and is impregnated after winding.

TABLE I

\begin{tabular}{lc} 
GEOMETRICAL DIMENSIONS OF RE-BCO ROEBEL CABLE \\
\cline { 1 - 1 } Number of tapes & 15 \\
Tape thickness & $150 \mu \mathrm{m}$ \\
Width: $\mathrm{W}$ & $12 \mathrm{~mm}$ \\
\hline Transposition pitch: $\mathrm{T}_{\mathrm{p}}$ & $226 \mathrm{~mm}$ \\
Crossing angle: $\alpha$ & $30^{\circ}$ \\
Straight part width: $\mathrm{w}_{\mathrm{l}}$ & $5.5 \mathrm{~mm}$ \\
Crossing part width: $\mathrm{w}_{\mathrm{c}}$ & $5.5 \mathrm{~mm}$ \\
\hline inner and outer radii not defined yet [13]
\end{tabular}

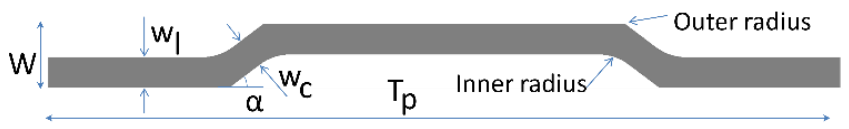

Fig.1. Dimensional parameters of a Roebel cable. 


\section{A. Magnet cross-section and magnetic field calculation}

Constrains due to Roebel cable mechanical behavior in the magnet ends - described in section III - drive the 2D crosssection design of the insert. For example, the upper-polar block seats at $\sim 60^{\circ}$ to avoid over bending in the magnet ends instead of $\sim 70^{\circ}$ as required for a good field quality [14] (see Fig. 2) - nevertheless, sextupolar and decapolar normalized field harmonics $\left(b 3=10^{4} \cdot \mathrm{B} 3 / \mathrm{Bl}\right.$ and $\left.b 5=10^{4} \cdot \mathrm{B} 5 / \mathrm{Bl}\right)$ due to the geometry are around one unit $\left(10^{-4}\right.$ of main field $\left.B 1\right)$ at two thirds of the aperture radius. It is worth noticing that magnetization current in Roebel cable may seriously worsen the magnetic field quality [15][16][17]. A small angular shim in the median plane is added to release some constrain in the bending of the upper cable of the median block (Roebel cables cannot be keystoned). Recent development on HTS tapes carried out at Bruker [18] may afford a powering of the insert in stand-alone up to $11,680 \mathrm{~A}$ corresponding to the $5 \mathrm{~T}$ central field target with $28 \%$ margin on the loadline. It corresponds to an overall current density in the insulated cable of $684 \mathrm{~A} / \mathrm{mm}^{2}$ and a tape engineering current density of 944 $\mathrm{A} / \mathrm{mm}^{2}$. In a $13 \mathrm{~T}$ background field, the insert can generate an extra $2.5 \mathrm{~T}$. Central and peak fields in the insert and outsert magnets are listed in Table II for the various operating conditions. In the worst case, the modification of the field configuration when energized as inside FRESCA2 would reduce its bursting forces by $2 \%$ and increase the vertical forces by $5 \%$ with no critical impact [19].

TABLE II

MAGNETIC AND MECHANICAL CONDITIONS IN THE MAGNETS

\begin{tabular}{lccc}
\hline & $\begin{array}{c}\text { Stand- } \\
\text { alone }\end{array}$ & $\begin{array}{c}\text { in FRESCA2 } \\
\mathbf{1 3} \mathbf{+ 2} \mathbf{~ T}\end{array}$ & $\begin{array}{c}\text { in FRESCA2 } \\
\mathbf{1 3}+\mathbf{2 . 5} \mathbf{~ T}\end{array}$ \\
\hline Current [A] & 11,680 & 6,100 & 7,670 \\
\hline Central field [T] & 5.01 & 15.01 & 15.52 \\
\hline Peak field insert [T] & 5.72 & $15.36^{* *}$ & $15.79^{* *}$ \\
\hline Peak field FRESCA2 [T] & N.A. & $12.93^{*}$ & $12.94^{*}$ \\
\hline $\begin{array}{l}\text { Insert azimuthal peak } \\
\text { stress [MPa] }\end{array}$ & 55 & 169 & 220 \\
\hline $\begin{array}{l}\text { Insert radial peak stress } \\
\text { [MPa] }\end{array}$ & 15 & 49 & 63 \\
\hline $\begin{array}{l}\text { Outer tube Von Mises } \\
\text { peak stress [MPa] }\end{array}$ & 120 & 385 & 490 \\
\hline
\end{tabular}

*13.13 T peak field in FRESCA2 powered at 10,660 A (no insert)

${ }^{* *}$ Peak field $1 \%$ higher in the S-shape cable exit (15.53 T and $\left.15.96 \mathrm{~T}\right)$.

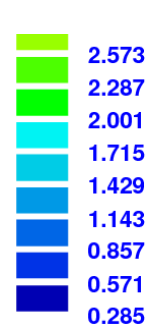

ROXIE $_{10.2}$

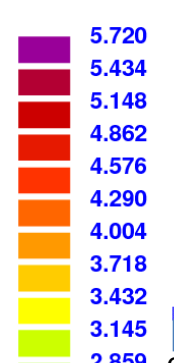

$2.8590 \mathrm{~mm}$

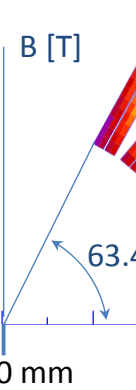

1

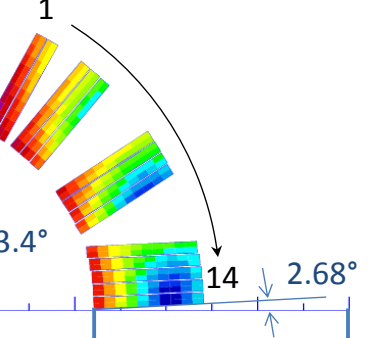

Fig.2. One fourth of the $\cos \vartheta$ dipole with the magnetic flux density in the 14 conductor turns.

\section{B. Mechanical analysis - standalone and insert modes}

A 2D finite element analysis of the mechanical structure - as defined in Fig. 3 - has been carried out with Cast3m [20]. A 14-mm-thick external shell minimizes the ovalization of the coil and leads to challenging transverse stress values up to $220 \mathrm{MPa}$ (see Table II). This value was thought to be bearable when first pressure tests on impregnated Roebel cables were carried out showing no performance degradation up to $250 \mathrm{MPa}$ [21]. Additional tests provided a lower value of $170 \mathrm{MPa}$ [22] that may limit the extra field of the insert to $2 \mathrm{~T}$ (see Fig.4). For more safety, another option is the use of an inner shell at the cost of a smaller aperture (Fig. 3 and 4). The part between the tubing and the coil is foreseen to be made of a polar pad and a stainless steel protection sheet (see Fig. 3). Steel properties are used in the mechanical model for both the shell and inner tube $(\mathrm{E}=210 \mathrm{GPa})$.
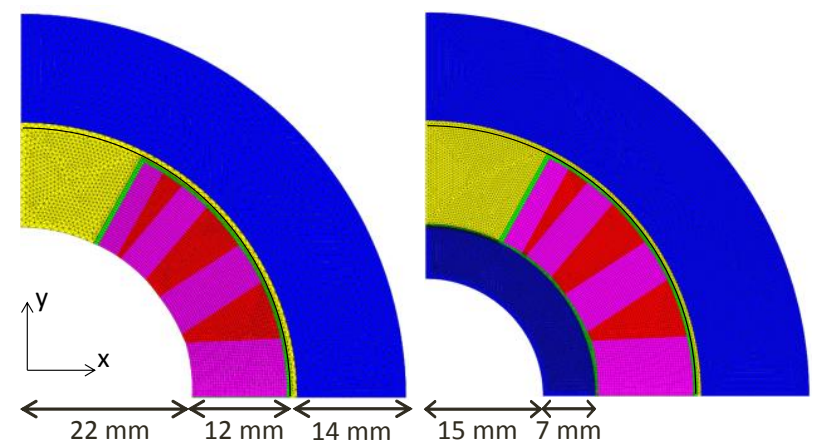

Fig. 3. Left: Mechanical structure with no inner shell and an aperture radius of $22 \mathrm{~mm}$. Right: Mechanical structure with a 7-mm inner shell and a radius aperture of $15 \mathrm{~mm}$.

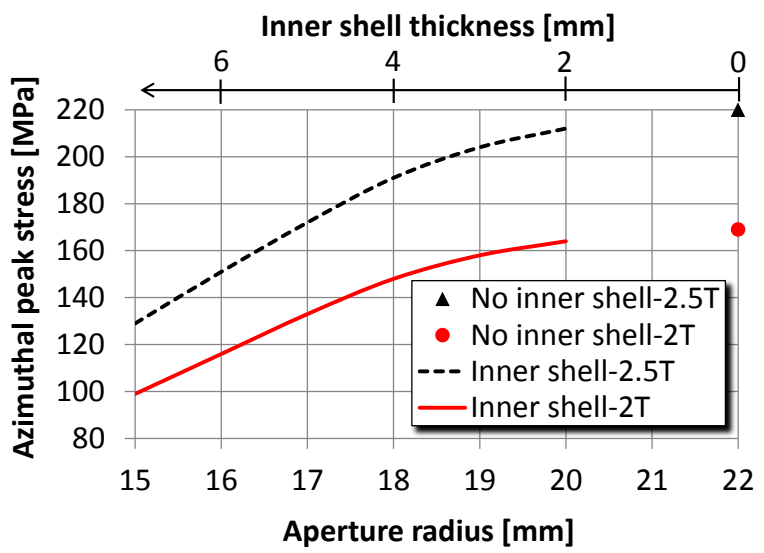

Fig. 4. Azimuthal peak stress in the coil [MPa] depending on the magnetic field conditions as well as the mechanical structure of the magnet. The baseline radius aperture is $20 \mathrm{~mm}$ and the maximum stress in the impregnated Roebel is $170 \mathrm{MPa}$.

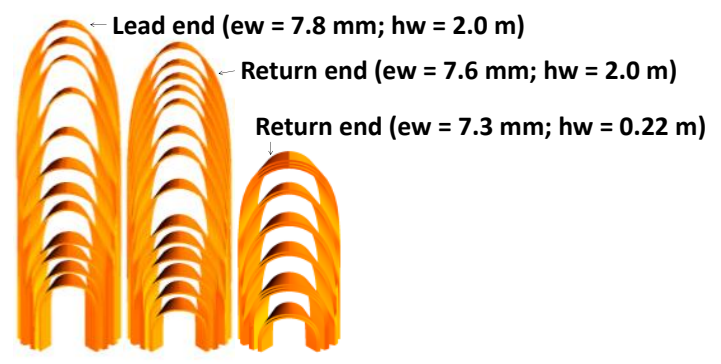

Fig. 5. In brackets are reported the minimal easyway (ew) and hardway (hw) for each head design. Left: Lead end with one spacer dedicated to each cable turn. Center: Return end with one spacer per turn, too. Right: End design by blocks of conductors. 


\section{3D DESIGN: ENDS AND CABLE EXIT}

\section{A. Optimization of Roebel cable mechanical constrains}

Initial minimum curvature radius on Roebel cable was set to 11-mm in easyway (ew, softway, out-of-plane) bending [23] and 2-meter in hardway ( $h w$, in-plane) bending. Therefore a first set of end spacers has been designed trying to locally follow these limits leading to one dedicated spacer per cable turn (Fig. 5 left) and a small median plane wedge (Fig. 2). For the innermost turns the easyway bending was as low as 7.6 $\mathrm{mm}$ due to the combination of the cos-theta layout and the small aperture [24]. It is worth noticing that $e w$ and $h w$ vary along the cable and that the minimum values reported in fig. 5 can occur only over a few millimeter length of cable. Therefore, the degradation may not be as severe as if the bending was over a couple of centimeters. In addition, the sponginess of the Roebel in the width direction can help to absorb some of the $h w$ bending. Following this paradigm, an additional return end set with less end spacers was designed and wound for testing. The end spacers were 3D printed by Fused Deposition Modeling (FDM) with 0.178-mm diameter filament. By means of this technology cheap models can be manufactured, mechanically resistant enough to be used in real winding tests.

\section{B. Winding tests}

Tests were carried out with a 10 -mm-wide dummy cable having a 426-mm transposition pitch. The cable was made of 15 tapes of $100-\mu \mathrm{m}$ in thickness. Tapes were punched and cable assembled at the Karlsruhe Institute of Technology. The thickness of the cable was about $0.9 \mathrm{~mm}$ which is slightly different in width and thickness from the baseline targets [9] (see Table I). The insulation of the cable is a fiber glass sleeve of $2 \times 125 \mu \mathrm{m}$ in thickness. A $0.3-\mathrm{mm}$ ribbon of mica is co-wound in an attempt to reach the $1.4-\mathrm{mm}$ nominal thickness of the baseline insulated cable. The remaining discrepancy of $2 \mathrm{~mm}$ in width, between the cable and the end spacer design, is considered negligible for the first winding tests. A low tension of $100 \mathrm{~N}$ is applied to the cable. The first turn is hold in place by means of a block of steel welded to the cable (in the final design the cable exit will help to keep the cable in place - see section below).

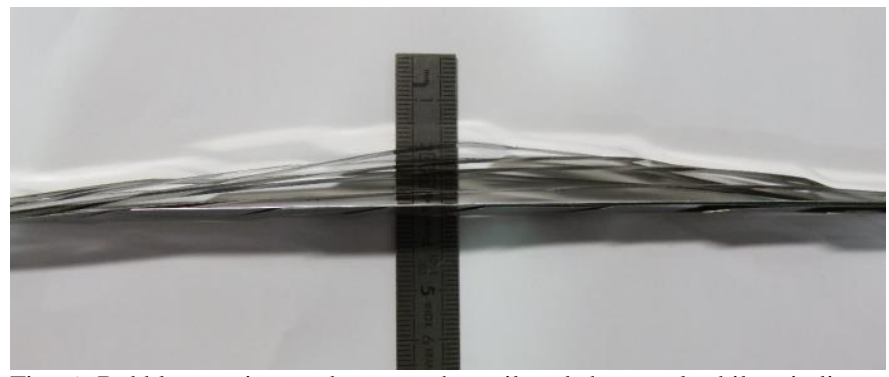

Fig. 6. Bubble popping up between the coil and the spool while winding a magnet. To avoid it the tapes need to be free of slipping at the cable end.

\section{1) Cable behavior while winding}

Roebel cables are made of tapes that must slip onto each other while winding due to the differential path of the tapes in the bent part of the magnet such as the ends [25]. The relative slippage leads to two main issues. On one hand, the nominal thickness of the whole cable, when the tapes are not evenly distributed, can increase. For example, the baseline bare cable of 1.2-mm-thick and made of $150-\mu \mathrm{m}$ tapes can become 1.35 -mm-thick at some locations ( $10 \%$ thicker $)$. This results in a bad Fleiter's surface [26] since the transverse force will be taken over these thicker locations leading to very high pressure spots. In addition, it may be difficult to fit all of the cable turns into the coil allocated space. On the other hand, if the relative slippage between two consecutive tapes becomes greater that the longitudinal gap, a bubble pops up. Indeed, at this point, the differential path cannot be absorbed anymore by the slippage along the cable. The tapes need to bulge in the direction perpendicular to the cable to compensate in a similar way of what is shown in figure 6 . That is certainly the main issue when winding Roebel cables. An increase of the transposition pitch of the cable and a decrease of the number of tapes in the cable are two options to fix it, both increasing the longitudinal gap of the cable. During the winding tests presently reported, the problem did not happen since the cable transposition pitch of 426-mm - almost twice as much as the baseline - was quite comfortable with respect to the number of tapes in the cable. Nevertheless, a bubble had to be handled between the coil and the spool while winding as shown in fig. 6 . The bubble is due to the fact that the cable is on a spool and the tapes not free of slipping at the end of the cable. As a drawback, in the extreme configuration, all the forces from the tensioner are taken by only one tape. Analysis showed that the maximal force that can be taken by a $100-\mu \mathrm{m}$ thick meander tape is about $150 \mathrm{~N}$ [27], this explains the small $100 \mathrm{~N}$ tension used in the tests.

\section{2) First test: one dedicated spacer per turn}

Figure 7 shows a picture of the final winding of the first test. The behavior of the cable was good in the magnet heads. Each cable turn seems to be in contact with the related end spacers as displayed in Fig. 8. The cable properly followed the mandrel curvature in the ends (see Fig. 9).

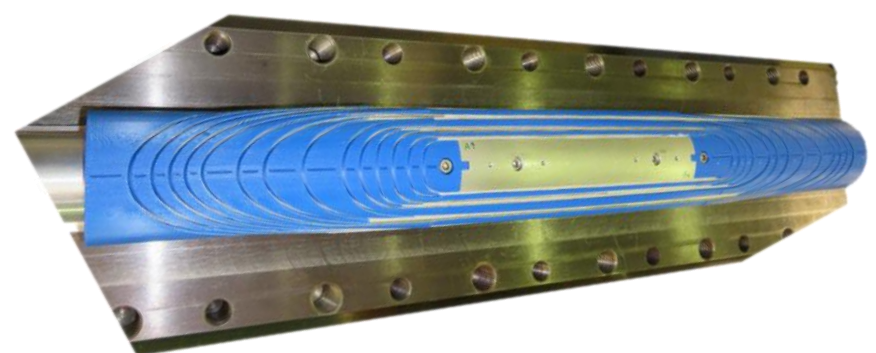

Fig. 7. Final winding with one spacer for each cable turn. The straight section is 200-mm-long.

The windings tests were successful and the design validated, nevertheless, the cost of real machining of the end spacers guided us to try a different design making use of less spacers (Fig. 5 right) and allowing for a longer straight section of the coil. The winding tests - at first on the return end only (Fig. 10) - were quite promising. Therefore, a modification of the $2 \mathrm{D}$ cross-section of the magnet with no median plane wedge has been planned. 


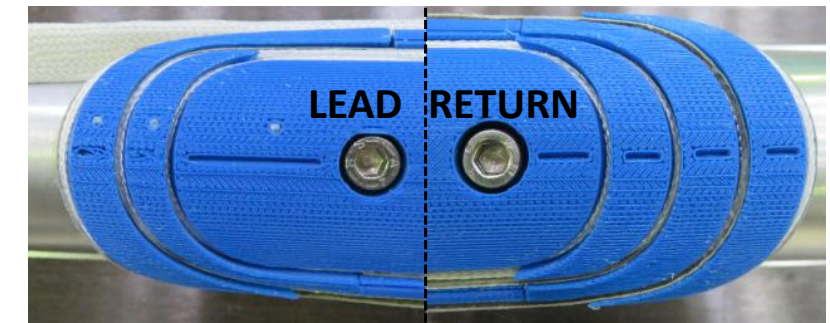

Fig. 8. First winding turns showing the position of the cable in-between the end spacers.

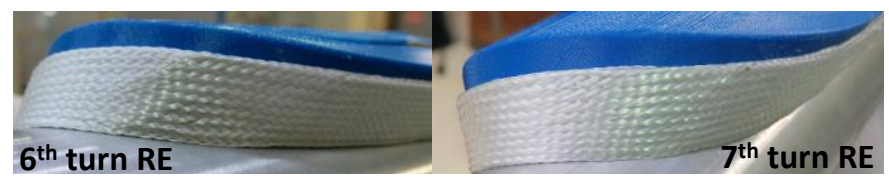

Fig. 9. Cable in contact with the mandrel at the end of two return end turns.

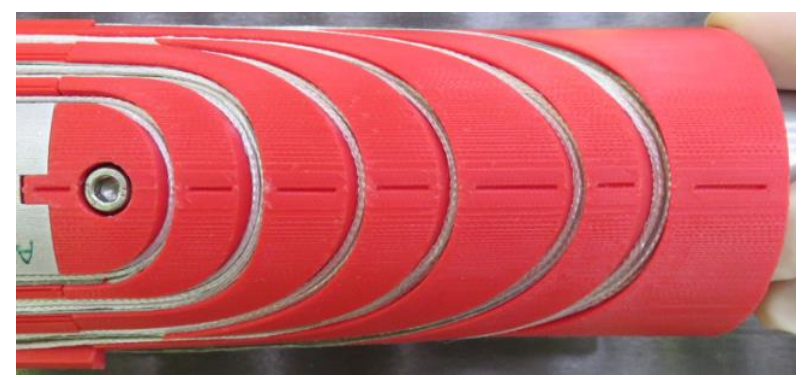

Fig. 10. Final winding of the return end of the design in blocks. The return head is about $130 \mathrm{~mm}$ to be compared to the $200 \mathrm{~mm}$ of the previous design.

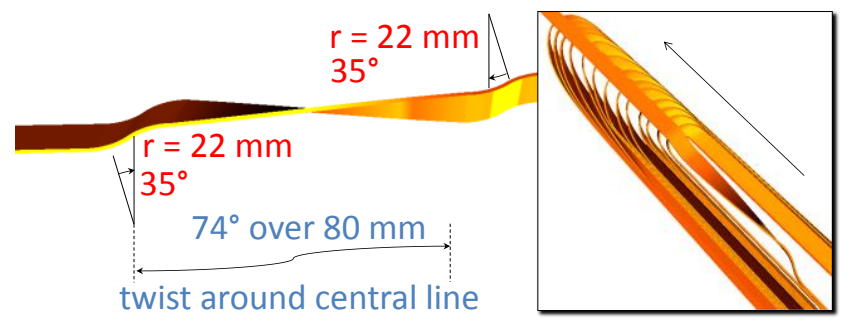

Fig. 11. Cable path outwards of the magnet made of a twist of $74^{\circ}$ over $80 \mathrm{~mm}$ in-between two S-shape easyway bendings with a radius of $22 \mathrm{~mm}$ and an opening angle of $35^{\circ}$.

\section{First turn path outwards of the magnet}

As the magnet consists of one conductor layer, the innermost turn of the coil needs to exit the magnet above the lead end as shown in Fig. 11. To do so, the cable follows a S-shape bending with a $22-\mathrm{mm}$ radius and an angle of $35^{\circ}$, then a twist of $74^{\circ}$ over $80 \mathrm{~mm}$ and a second and similar S-shape bending. The strain generated by such a twist on a 12 -mm wide tape is ranging from $-0.16 \%$ to $+0.31 \%$ from the center of the tape to its edge which is safe [28]. This result is conservative since the meander tapes of the Roebel should not experience such a strain. A test in a 3D printed mold was done and showed the feasibility of shaping the Roebel cable as previously described. In the mold the groove in which the cable was set had a $0.1-\mathrm{mm}$ gap around the insulated cable. As the coil will eventually be impregnated the gap was increased to $0.2-\mathrm{mm}$ in the final design of the magnet components (Fig. 12) to get more flexibility to insert the cable.

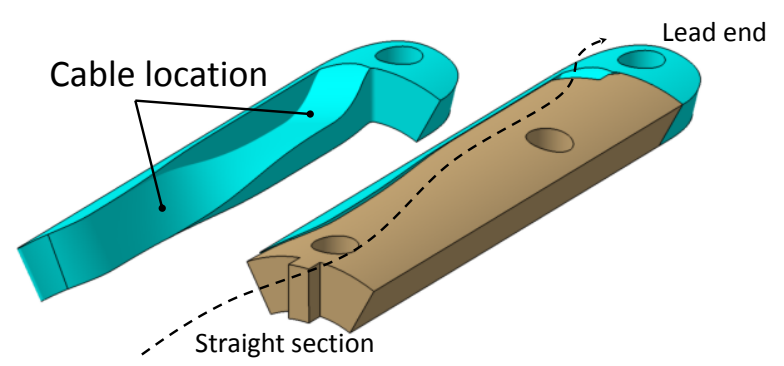

Fig. 12. Magnet components smoothly shaping the cable coming from the first turn of the straight part (turn \#1 in Fig.2) to exit the magnet above the lead end turns. The total length is about $130 \mathrm{~mm}$.

\section{CONCLUSION}

An electromagnetic and mechanical analysis of a $\cos \vartheta$ dipole based on a Roebel cable technology has been conducted. The design of the magnet is driven by the dimension of the outsert magnet, namely FRESCA2, and by the electromechanical resilience of the cable: i) transverse pressure leading to the use of an inner shell in a single layer magnet, ii) out-of-plane bending impacting the upper block position in the 2D crosssection, iii) in-plane bending ruling the 3D end design and iv) twisting governing the length of the cable exit components.

Winding tests have been carried out to check the behavior of the cable in the different sections of the magnet. They showed that the cable tapes need to slide on top of each other. Indeed, the longitudinal gap of the cable must be long enough to absorb the tape slippage and avoid any bulging effect. In addition, the slippage of the tapes can increased the nominal thickness of the cable by the thickness of one tape.

Further tests are foreseen with a dummy baseline cable to mechanically validate all the coil components and to check the windability with a small transposition pitch of $226 \mathrm{~mm}$. Twisting tests of HTS Roebel cable are truly considered to better understand the impact of the cable exit on the cable critical current performance.

\section{ACKNOWLEDGEMENTS}

The authors would like to thanks S. Izquierdo-Bermudez from Cern for discussion about Roxie software as well as, from CEA, A. Przybylski, S. Somson, J.J. Goc and P. Leveque for winding test and $\mathrm{A}$. Acker for magnet component drawings.

\section{REFERENCES}

[1] L. Bottura, "The FCC magnet program: challenges and opportunities for HTS", $1^{\text {st }}$ Workshop on Accelerator Magnets in HTS, DESY, Hamburg, Germany, $21^{\text {st }}-23^{\text {rd }}$ May 2014.

[2] G. de Rijk, "The EuCARD high field magnet project", IEEE Applied Superconductivity, vol 22, 3, June (2012).

[3] L. Rossi et al., "The EuCARD-2 Future Magnets project: the European collaboration for accelerator quality HTS magnets", IEEE Applied Superconductivity, vol 25, 3, June (2015).

[4] L. Rossi et al., "Status of the Demonstrator Magnets for the EuCARD-2 Future Magnets Project", European Conference on Applied Superconductivity, Lyon, France, $6^{\text {th }}-10^{\text {th }}$ September 2015.

[5] F. Borgnolutti et al. "Fabrication of 5.4 T dipole insert magnet using REBCO tapes", International Conference on Magnet Technology, Seoul, South Korea, $18^{\text {th }}-23^{\text {rd }}$ October 2015.

[6] J. Himbele et al., "HTS dipole magnet for a particle accelerator using a twist stack cable", European Conference on Applied Superconductivity, Lyon, France, $6^{\text {th }}-10^{\text {th }}$ September 2015. 
[7] G. Kirby et al., "Accelerator quality HTS dipole magnet demonstrator designs for the EuCARD-2, 5T $40 \mathrm{~mm}$ clear aperture magnet", IEEE Applied Superconductivity, vol 25, 3, June (2015).

[8] G. Kirby et al., "Detailed development of a REBCO superconducting coil using Roebel $10 \mathrm{kA}$ class cable at $4 \mathrm{~K}$ operation", International Conference on Magnet Technology, Seoul, South Korea, $18^{\text {th }}-23^{\text {rd }}$ October 2015.

[9] C. Lorin, M. Segreti at al, "Cos- $\theta$ design of dipole inserts made of REBCO-Roebel or BSCCO-Rutherford cables", IEEE Trans. on Applied Superconductivity, vol. 25, n³, pp4000305, (2015).

[10] P. Ferracin et al. "Development of the EuCARD $\mathrm{Nb}_{3} \mathrm{Sn}$ dipole magnet FRESCA2", IEEE Trans. on Applied Superconductivity, vol. 23, n³, pp4002005, (2013).

[11] W. Goldacker et al., "Roebel cables from REBCO coated conductors: a one-century-old concept for the superconductivity of the future" Superconductor Science and Technology, vol 27, 9, (2014)

[12] L. Bottura et al., "Advances in the Development of a 10-kA Class REBCO cable for the EuCARD2 Demonstrator Magnet"

[13] C. Barth et al, ., "Electromechanical analysis of Roebel cables with different geometries" Superconductor Science and Technology, vol 25, 025007, (2015)

[14] L. Rossi, E. Todesco, "Electromagnetic design of superconducting dipoles based on sector coils", Phys Rev STAB, 10, 112401, (2007)

[15] V. Zermeno, F. Grilli, F. Sirois, "A full 3D time-dependent electromagnetic model for Roebel cables" Superconductor Science and Technology, vol 26, 052001, (2013)

[16] S. Terzieva et al. "Transportation and magnetization ac losses of ROEBEL assembled coated conductor cables: measurements and calculations" Superconductor Science and Technology, vol 23, 010423, (2010)

[17] L. Bottura et al., "Measured and modelled AC magnetization loss of the REBCO Roebel baseline cable for the EUCARD2 accelerator insert magnet", International Conference on Magnet Technology, Seoul, South Korea, $18^{\text {th }}-23^{\text {rd }}$ October 2015.

[18] A. Usoshin, "Development and processing of high-field HTS coated conductors for EUCARD2 project",WP10 meeting at Cern, January $8^{\text {th }}$ 2015 (https://indico.cern.ch/event/354955/)

[19] P. Ferracin, private communication, 28 July 2015.

[20] P. Verpaux, T. Charras, and A. Millard, "Castem 2000 une approche moderne du calcul des structures", Calcul des structures et intelligence artificielle, pp. 261-271, 1988.

[21] S. Otten, "Transverse pressure dependence of the critical current in epoxy impregnated REBCO Roebe cables", Master thesis, University of Twente, (October 10, 2014)

[22] S. Otten et al. "Increase of tolerance for transverse loading at REBCO Roebel cables with impregnation" European Conference on Applied Superconductivity, Lyon, France, $6^{\text {th }}-10^{\text {th }}$ September 2015.

[23] W. Goldacker et al., "Roebel assembled coated conductors (RACC): Preparation, properties and progress," IEEE Trans. on Applied Superconductivity, vol. 17, no. 2, pp. 3398-3401, Jun. 2007.

[24] C. Lorin, "Cos $\theta$ magnet design option", EuCARD2 report, CERN-ACC2015-0043, pages 32, (2015)

[25] J. Fleiter, C. Lorin et al., "On Roebel cable geometry for accelerator magnets" European Conference on Applied Superconductivity, Lyon, France, $6^{\text {th }}-10^{\text {th }}$ September 2015

[26] J. Fleiter et al. "Characterization of Roebel cable for potential use in high-field magnets" Transactions on Applied Superconductivity, vol. 25, 3, 4802404, (2015).

[27] J. Murtomaki, "Roebel strand max acceptable tension" CERN Technical note 2015-25,1532170 (2015)

[28] M. Takayasu, L. Chiesa, L. Bromberg, J.V. Minervini, "HTS twisted stacked-tape cable conductor" Superconductor Science and Technology, vol 25, 014011, (2012) 